\title{
A study that shows nothing
}

\author{
Cristian Baicus
}

Received: 3 March 2012/ Accepted: 8 March 2012/Published online: 21 March 2012

(C) Springer-Verlag 2012

Sir,

In their study concerning obesity and coronary risk in patients treated with second-generation antipsychotics, Correll et al. [1] wanted to see whether obesity is an independent risk factor for the 10-year risk of coronary heart disease calculated by Framingham method. After excluding the patients with metabolic syndrome and those taking antihypertensive, hypoglycemic, and lipid-lowering drugs, they discovered that "the 10-year risk of coronary artery disease events was very low and virtually identical in the obese and normal weight patients" and, therefore, "obesity does not appear to be an independent predictor for the 10-year risk of coronary heart disease events in patients without metabolic syndrome treated with second-generation antipsychotics."

However, I think there are two problems concerning the study design and conclusion:

First, the chosen outcome (risk estimation by the Framingham method) does not permit to evaluate obesity as an independent risk factor: suppose the risks had been different in obese and nonobese patients, obesity would have been an confounder, and not an independent risk factor, because it would have increased risk through age, gender, blood pressure, smoking, and plasma levels of total and high-density lipoprotein cholesterol, which were used to compute the Framingham score. In fact, the authors proceeded as they would have wanted to exclude obesity as an independent risk factor, because they stratified by it.

Second, excluding the patients with metabolic syndrome and those taking antihypertensive, hypoglycemic, and lipid-lowering drugs, therefore keeping only patients with normal constituents of Framingham score, the investigator had not any chance to find a difference between obese and nonobese patients. A flowchart of the study would have been useful, in order to see whether the excluded patients were not predominantly obese, as probably happened.

Conflict of interest The author declares that he has no conflict of interest.

\section{Reference}

1. Correll CU, Kane JM, Manu P (2011) Obesity and coronary risk in patients treated with second-generation antipsychotics. Eur Arch Psychiatry Clin Neurosci 261:417-423
C. Baicus $(\bowtie)$

Department of Internal Medicine, Colentina University

Hospital, Carol Davila University of Medicine, Soseaua Stefan

cel Mare 19-21, Sector 2, 020125 Bucharest, Romania

e-mail: cbaicus@clicknet.ro

C. Baicus

Clinical Research Unit RECIF (Réseau d'Epidémiologie

Clinique International Francophone), Bucharest, Romania 